\title{
Single-Region Robust Perimeter Traffic Flow Control
}

\author{
Balázs Kulcsár, Konstantinos Ampountolas and Azita Dabiri
}

\begin{abstract}
In this paper, we propose a two-stage robust perimeter flow control policy to prevent congestion in singleregion transport networks. We describe the single-region traffic dynamics by utilising the so-called Macroscopic or Network Fundamental Diagram (MFD or NFD), a nonlinear relation between network-wide mean flow and accumulation of vehicles. By using MFD relaxation conditions and structuring uncertainties, we reformulate the nonlinear flow dynamics to set of uncertain Linear Parametrically Varying (LPV) ones.

In controller design stage one, we apply the concept of a two degree of freedom, induced $\mathcal{L}_{2}$ norm minimising LPV controller. Within the generalised performance output, we include tracking performance to follow a pre-defined critical accumulation in uncertain model environment. Output feedback LPV controller ensures generalised disturbance attenuation conditions with appropriate gated input flow at the perimeter.

Second, an optimal quadratic control allocation algorithm is employed to distribute the ordered flow to entrance link green stages (flow equivalent green time) in a number of candidate junctions at the perimeter of the network. The constrained allocation techniques complement the robust controller enabling real-time applicability of the proposed methodology.

Finally, the proposed robust control scheme is tested and compared with a reference tracking bang-bang controller in macroscopic simulation. Results indicate the viability of the proposed LPV controller and its rapid and accurate tracking behaviour under highly uncertain parameters of the MFD.
\end{abstract}

\section{INTRODUCTION}

In recent years, a promising approach to address specific classes of nonlinear control problems has been developed, i.e. the Linear Parameter Varying (LPV) systems in statespace, see e.g. [1], [2]. In LPV techniques, controller design is triggered by a real-time available scheduling parameter vector. Linearity represents the casual structure of the dynamic problem within the state space and control input variables. Although LPV description preserves the linear structure, coefficient matrices are changing, scheduled by the parameter vector. In a particular case when the parameter vector coincides (partially or entirely) with the state vector the system is called quasi-Linear Parameter Varying (qLPV) system. The LPV framework has been already successfully applied to various problems; including freeway traffic flow for local throughput maximisation [3], [4]. In this work, the robust LPV controller framework is adopted to the problem

B. Kulcsár and A. Dabiri are with Department of Signals and Systems, Chalmers University of Technology, Hörsalsvägen 9-11, Gothenburg, SE412-96, Sweden, \{kulcsar, azitad\}@chalmers. se. They gratefully acknowledges the support of Chalmers' new initiative in transportation, the Transport Area of Advance.

$\mathrm{K}$. Ampountolas is with the School of Engineering, University of Glasgow, Glasgow G12 8LT, UK, Konstantinos.Ampountolas@glasgow.ac.uk. of optimal perimeter flow control for single-region networks described by macroscopic traffic flow models.

A macroscopic model of urban traffic was proposed in [5], further developed in e.g. [6], [7] and fitted to experimental data in [8], [9], [10] and others. This model is the so-called Macroscopic Fundamental Diagram (MFD) of urban road networks; it presumes (under certain regularity conditions) that traffic flows dynamics could be treated macroscopically as a single-region dynamic system with vehicle accumulation $n$ as a state variable. The main feature of an MFD is that for a critical accumulation $\hat{n}$ flow capacity is reached, although recent works with experimental data (see e.g. [10]) have shown that capacity may be observed over a range of vehicle accumulation-values. This property gave rise to perimeter flow control to improve mobility in single-region homogeneous [11], [12], [13] or multi-region heterogeneous cities [14], [15], [16]. Perimeter flow control "meters" the input flow to the system and hold vehicles outside a controlled area if necessary, so as to maximise the throughput.

Recent works have identified that the shape of an MFD is affected by different factors, including the spatial distribution of congestion [17], traffic-responsive signal control [18], [19], [10], traffic (mode) composition [20], loop-detector placement [21], non-recurrent day-to-day traffic patterns [10], etc. Moreover, flow capacity in urban networks may be observed over a range of vehicle accumulation-values, and thus the critical accumulation cannot accurately be specified [10]. Recently developed feedback perimeter flow control strategies rely mostly on nominal pre-specified set points $\hat{n}$ and/or prone to the accuracy of a linearised nominal [11], [12], [15] or linearised uncertain dynamics [13].

In this line, the paper addresses the problem of robust perimeter control with well-posed uncertainty traffic flow description. Rather than using the nonlinear modelling framework, herewith an affine and quasi parametrisation of the flow dynamics scheduled in vehicle accumulation is introduced. Within the LPV framework, we structure mismatches in order to capture MFD parametric, input model and reference uncertainties. By complementing the uncertain traffic flow dynamics with additional dynamic weights, we include all plausible disturbance/uncertainty related information. This augmented system forms a basis for robust LPV controller design, for induced $\mathcal{L}_{2}$ norm minimising dynamic controller synthesis. Hard physical input constraints connected to green stages are included as a separate design condition with constrained controller allocation problem.

The rest of the paper is organised as follows. Section II presents the parameter-dependent modelling of the MFD. 
Section III, we briefly introduce the goal of robust LPV controller design and optimal allocation of the ordered flow to the perimeter. In Section IV, a case study for the San Francisco network illustrates the robustness of the proposed scheme. Conclusions and further research directions are drawn in Section V.

\section{Parameter-Dependent Traffic Flow Model for Single-Region Perimeter CONTROL}

Consider a single-region homogeneous network with $n(t)$ be the accumulation of vehicles at time $t \geq 0$. Let $Q_{c}(n(t))$ $(\mathrm{veh} / \mathrm{h})$ be the total regional circulating flow as it can be estimated by Edie's generalised definition of flow, i.e., weighted average of link flows with link lengths. If we assume that the average trip length $L$ in the network is constant and the average link length is given by $l$, then the output (throughput) of the network can be expressed as $Q(n(t))=(l / L) Q_{c}(n(t))$ [11]. Output $Q$ (called MFD or exit function) is the estimated rate (flow) at which vehicles complete trips per unit time either because they finish their trip within the network or because they move outside the network. This function describes steady-state behaviour of single-region homogeneous networks if the input to output dynamics are not instantaneous and any delays are comparable with the average travel time across the region.

Assumption 1: If the network exhibits a consistent MFD $Q_{c}$ then output $Q$ describes the behaviour of the singleregion system when it evolves slowly with time.

In view of Assumption 1, the dynamics of the singleregion system are described by the following ordinary nonlinear differential equation

$$
\frac{d n(t)}{d t}=\beta(t)-Q(n(t))+d(t), \quad t \geq 0
$$

where $\beta(t)$ is the input (controlled at the perimeter) flow to the region, $Q(n(t))$ is the regional total outflow (the MFD), and $d(t)$ is the uncontrolled traffic demand (disturbances).

Assumption 2: Output $Q(n(t))$ is a $n_{Q}$ times differentiable function of vehicle accumulation $n$ over the bounded range of accumulation, $\underline{n} \leq n(t) \leq \bar{n}$.

In view of Assumption 2, a polynomial in the vehicle accumulation $n$ form of $Q$ is explicitly considered and gives rise to parametrisation. More precisely, we assume the existence of a nominal and centred $(Q(0)=0)$ fundamental relationship given by

$$
Q(n(t))=\sum_{i=1}^{n_{Q}} c_{i} n^{i}(t)
$$

where $n_{Q}$ is a pre-specified MFD parametrisation degree of freedom and $c_{i}$ are coefficients. Such nominal relationships can be estimated by Edie's generalised definition of flow under the assumption that demand is spatially homogeneous.

In most of the cases, to yield a nominal MFD analytical form like (2), some approximation is involved. For instance, Taylor's theorem can be applied, introducing truncation error. Despite the findings of a nominal MFD, these curves should not be a universal law, as discussed in Section I.
Capacity flow and critical accumulation of the MFD can be affected by the spatiotemporal distribution of congestion in the network, the applied traffic-responsive signal control, traffic composition (e.g. cars vs. buses), non-recurrent dayto-day traffic patterns, or the spatial placement of sensors. Therefore, a nominal MFD curve can only be valid under certain conditions. Instead of using a single MFD curve, we hereby propose to use a set of uncertain but well-structured relationship.

Proposition 1: The relative uncertainty structure of MFD is given by a set of relationship as follows

$$
\tilde{Q}(n, c, \delta)=(\sum_{i=1}^{n_{Q}} \underbrace{c_{i}\left(1+\delta_{i}(t)\right)}_{\tilde{c}_{i}} n^{i-1}(t)) \cdot n(t)
$$

where $\left|\delta_{i}\right|<1, i=1, \ldots, n_{Q}$ are real valued parametric uncertainty coefficients and $c_{i}$ are the nominal MFD parameters as in (2).

In (3), we define relative coefficient wise polynomial uncertainties $\tilde{c}_{i} \leq \tilde{c}_{i} \leq \overline{\tilde{c}}_{i}, i=1, \ldots, n_{Q}$ with respect to the nominal MFD parameters $c_{i}$. Introducing the set of models offered in Proposition 1 to (1), i.e. the structured uncertain MFD form with scheduled in vehicle accumulation, the single-region system dynamics can be written as

$$
\begin{aligned}
\frac{d n(t)}{d t} & =\beta(t)-\left(\sum_{i=1}^{n_{Q}} c_{i}\left(1+\delta_{i}(t)\right) n^{i-1}(t)\right) \cdot n(t)+d(t) \\
& =A(p(t)) n(t)+B_{2} \beta(t)+B_{1}(p(t)) \tilde{d}(t)
\end{aligned}
$$

where $p(t)=\left[\begin{array}{lllll}1 & n(t) & n^{2}(t) & \cdots & n^{n_{Q}}(t)\end{array}\right]^{T} \in \mathcal{P}$ is a vector of scheduling parameters that are assumed measured in real-time; $\tilde{d}(t)=\left[\begin{array}{lllll}d(t) & d_{\beta}(t) & \delta_{1}(t) & \cdots & \delta_{n_{Q}}(t)\end{array}\right]^{T}$ is a vector of disturbances and parameter uncertainty coefficients, where $d_{\beta}(t)$ is input uncertainty for vehicles to approach the network from the perimeter. Clearly $B_{2}=1$, $A(p(t))$ is a scalar depending on $p(t)$, and $B_{1}(p(t))$ is a row vector consisting of columns triggered by $p(t)$. Introducing $\tilde{Q}$, the nonlinear flow model (1) is reformulated into a set of uncertain and LPV, where the model description is scheduled by a measurable state variable $n(t)$. In the LPV model (4), the polynomial MFD decomposition gives rise to the existence of basis functions and hence to an affine in the scheduling parameter form. In other words, the model (4) is a linear state-space model with a scheduling dependency in the coefficient matrices. As long as the polynomial MFD model is exact, the LPV model transformation is exact in view of the original nonlinear model (1).

Problem 1: Based on the previous LPV flow model, our goal is to devise a two-step perimeter traffic flow control scheme as follows:

- Scheduled by $n(t)$, design a robust controller that attenuates unknown disturbances and tracks uncertain critical vehicle accumulation $\hat{n}(t)$ by means of changes the gated input flow $\beta(t)$, i.e. robust throughput maximization.

- Develop a systematic approach for distributing the obtained input flow $\beta(t)$ to a number of signalised junctions 
located at the perimeter of the network in an constrained and optimal way.

\section{Robust Perimeter Control Design}

A two degree of freedom reference tracking LPV control policy depicted in Fig. 1 is employed to address Problem 1.

\section{A. Robust LPV Control for Perimeter Traffic Flow Control}

For robust controller design, we first augment the transformed open loop LPV model (4) with additional dynamic weightings [22] in order to include all a-priori performance and disturbance scaling information (weight selection procedure is described in Section IV-B). We classify the inputs as controlled (gated) flow and uncontrolled disturbances, and the outputs as performance and measured ones. The continuous time state-space representation of the augmented system is given by

$$
\left[\begin{array}{l}
\dot{n}(t) \\
z(t) \\
y(t)
\end{array}\right]=\left[\begin{array}{ccc}
A(p(t)) & B_{1}(p(t)) & B_{2} \\
C_{1}(p(t)) & D_{11}(p(t)) & D_{12}(p(t)) \\
1 & D_{21}(p(t)) & 0
\end{array}\right]\left[\begin{array}{c}
n(t) \\
d(t) \\
\beta(t)
\end{array}\right]
$$

where $y(t)=n(t)+d_{n}(t)$ is the measured state output with noise $d_{n}(t)$ imposed by the sensors (loop-detectors). Redefine $d(t)=\left[\begin{array}{lll}\tilde{d}(t)^{T} & d_{n}(t) & r(t)\end{array}\right]^{T}$ where $r(t)=\hat{n}(t)$ is the reference critical vehicle accumulation to track. The following performance vector is defined with appropriate dynamic weightings $z(t)=\left[\begin{array}{llll}z_{\delta}^{T} & z_{r} & z_{\beta}^{T} & z_{e}\end{array}\right]^{T}$, where $z_{\delta}$ refers to uncertainties in MFD (3), $z_{r}$ denotes the uncertainty in the reference signal (since $\hat{n}(t)$ is not exactly known), the vector $z_{\beta}$ comprises both input performance and input multiplicative uncertainty, and $z_{e}$ describes tracking error performance. Note that the state, control, performance and output matrices (with appropriate dimensions) in (5) are functions of the $p(t)$.

Formally, we intend to minimise the induced norm between the generalised disturbance vector $d$ and the qualitative output $z$ (2-norm signals) as,

$$
\left\|T_{z d}(p)\right\|_{2}=\sup _{p \in \mathcal{P}} \sup _{\|d\|_{2} \neq 0} \frac{\|z\|}{\|d\|} \leq \gamma
$$

where $T_{z d}$ is the transfer function matrix from $d$ to $z$ and $\gamma>0$ denotes the disturbance attenuation level.

Remark 1: Although state feedback perimeter control is proposed, this policy can be seen as dynamic output feedback scheme due to the added weighting dynamics.

Thus the controller takes the compact form

$$
\left[\begin{array}{c}
\dot{x}_{c}(t) \\
\beta_{c}(t)
\end{array}\right]=\left[\begin{array}{ll}
A_{c}(p(t)) & B_{c}(p(t)) \\
C_{c}(p(t)) & D_{c}(p(t))
\end{array}\right]\left[\begin{array}{c}
x_{c}(t) \\
\tilde{y}(t)
\end{array}\right]
$$

where $x_{c}$ and $\beta_{c}$ are the controller's state and the gated flow input, with $\tilde{y}(t)=[r(t) y(t)]^{T} . A_{c}, B_{c}, C_{c}, D_{c}$ are the controller's parameter dependent coefficient matrices with appropriate dimensions. If feasibility conditions are met, the LPV controller is quadratically (exponentially) stable with $\gamma>0$ disturbance rejection level. The controller's state-space matrices can be obtained as the solution of Linear Matrix Inequalities (LMI).To derive the closed-loop controller (7), grid based LPV techniques are applied over a finite number of LMIs with the help of the LPVTools [23]. Note, scheduling parameter rate information might be included into the design phase $\dot{p} \leq \dot{p}(t) \leq \overline{\dot{p}}$ to result in a less conservative $\gamma$, where $\dot{p}$ and $\overline{\dot{p}}$ are the minimum and maximum allowed bounds.

Remark 2: As in [22], a scheduling saturation parameter is introduced to handle ordered input flow saturation.

Remark 3: Parameter rate bounds can be obtained from the slope of the tangent line of free-flow speed and "jam" accumulation speed.

\section{B. Optimal Flow Allocation to Green Time}

In subsection III-A $\beta(t)$ gated flow is computed by means of handling uncertainties and disturbances in the perimeter flow dynamics. Thus, we only weight/scale the gated input flow without taking into account saturation flow and entrance link green stage constraints imposed during the deployment phase at the perimeter of the network.

At the deployment phase the ordered flow $\beta$ is transformed to entrance link green stages in a number of (pre-specified) junctions at the perimeter of the network. On the other hand, link green time is subject to minimum and maximum bounds with given cycle times. Instead of directly incorporating these physical constraints into the robust controller synthesis, a two-step procedure is employed as described in Problem 1. To this end, the ordered flow $\beta(t)$ obtained from the robust LPV controller (7) is distributed to equivalent entrance link green stages at the perimeter with the help of a controller allocation method [24] as follows. Consider a number $n_{j}$ of candidate signalised junctions at the perimeter with (not necessary constant, but identical) cycle times $T_{c i}$ and entrance link saturation flow rates $s_{i}$ (for all lanes). Denote $\mathcal{T}$ the green time vector with elements $\tau_{i}$, which are subject to minimum (usually non-zero to avoid long queues and delays at the perimeter of the network) and maximum bounds. These constraints may be brought to the general form $A \mathcal{T} \leq B$. To yield optimal green times, the following minimisation problem is solved

$$
\begin{aligned}
& \min _{\mathcal{T}}\left(\beta(t)-\sum_{i=1}^{n_{j}} r_{i} \frac{s_{i}}{T_{c i}} \tau_{i}\right)^{2} \\
& \text { subject to } \\
& A \mathcal{T} \leq B
\end{aligned}
$$

where $r_{i}$ is a pre-specified constant scaling weight (e.g. to prioritise junctions or entrance links). In fact the ordered flow $\beta(t)$ is $\ell_{2}$ optimally allocated to a fixed set of green times by using a zero-order hold with sampling $T_{c i}$. The green time split procedure might result error since expression (8) is a weighted constrained least-squares problem with error term $\epsilon(t)=\beta(t)-\hat{\beta}(t)$, where $\hat{\beta}(t)$ is the (real) gated flow implemented to the junctions. Finally, the optimisation (8) ensures the best possible constrained green time allocation that minimises the quadratic flow deviations with respect to $\beta(t)$. 


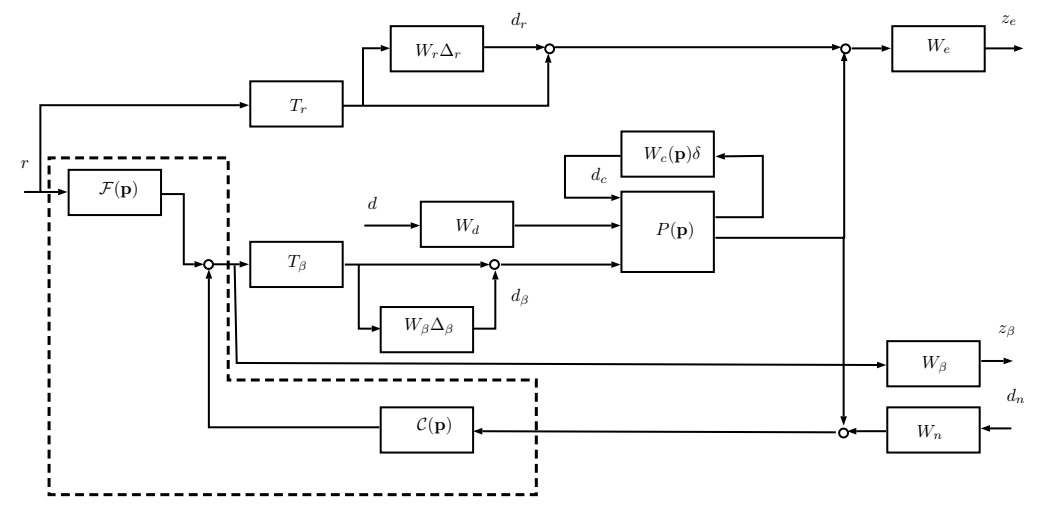

Fig. 1. Weighted system interconnection for controller design. $P(p)$ depicts the block for the nominal uncertain flow model given in Proposition 1, $\mathcal{F}(p), \mathcal{C}(p)$ denote the controller blocks, while $\Delta$ blocks are norm bounded uncertainties; $W_{\star}$ is standing for known input/performance weights; and $T_{\star}$ are nominal transfer function information added.

Remark 4: In fact, input saturation constraints are separately handled by control allocation method in a "static" way. In subsection III-A the ordered flow $\beta$ might violate green stages related constraints, that is forced to satisfy in the control allocation independently. This might result in performance degradation by input flow mismatch, that is only indirectly considered in this paper (input multiplicative uncertainty at the plant control input) ${ }^{1}$.

\section{CAse Study AND Results}

\section{A. Case Study Description}

Fig. 2 depicts the shape of $Q_{c}$ in function of $n(t)$ for a 2.5 square mile area of Downtown San Francisco, CA, including 100 junctions and 400 links. Fig. 2 confirms the existence of an MFD like-shape for the area, which shape is seen to depend on the accumulation of vehicles. It can be seen that as the vehicle accumulation is increased from zero, the network flow increases to a maximum (flow capacity) and then turns down and decreases sharply to a low value possibly zero (in case of gridlock). Flow capacity (around $30 \cdot 10^{4} \mathrm{veh} / \mathrm{h}$ ) is observed at a vehicle accumulation of $6000 \mathrm{veh}$. The shape of the MFD (and its critical parameters) was reproduced under different demand and OD scenarios (with Dynamic Traffic Assignment activated to capture somewhat adaptive drivers) in a microsimulation study with AIMSUN [15]. The shape of $Q_{c}$ (or $Q$ ) can be captured by different functions, e.g. quadratic or exponential. More precisely, the data of Fig. 2 can be approximated by the following 2 nd order polynomial:

$$
Q_{c}(n)=-0.0066 n^{2}+87.408 n
$$

where $n \in[0,12000]$. To derive the output $Q$ from $Q_{c}$ an average trip length $L=1.75 \mathrm{~km}$ and average link length $l=0.25 \mathrm{~km}$ were considered. The value of $L$ is consistent with the average trip length and the travel time across the test area of San Francisco. The nominal vehicle accumulation for (9) is found $\hat{n}=6612$ veh.

\footnotetext{
${ }^{1}$ Robust LPV model predictive control or constrained robust LPV techniques can be applied on the expense of significantly higher computational burden. Furthermore, other than quadratic control allocation methods can also be applied.
}

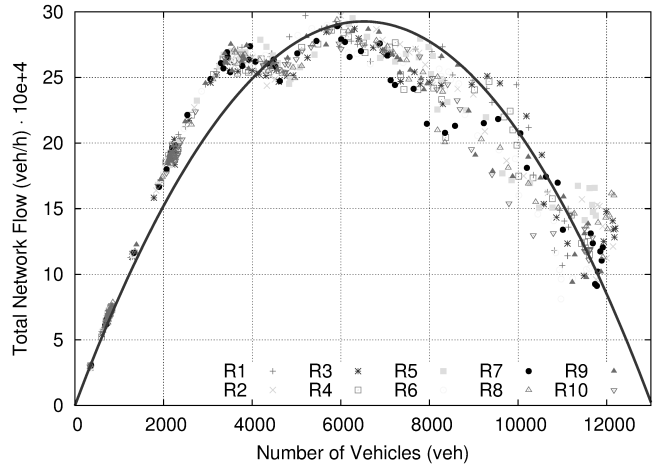

Fig. 2. Fundamental diagram $Q_{c}$ for Downtown San Francisco, CA [15].

\section{B. Weight Selection Procedure and Controller Design}

Fig. 1 depicts the system interconnection of a two-degree of freedom reference tracking control scheme. Both dynamic and static weightings are proposed to complement the nominal model information subject to disturbance characterisation and performance requirements. In the sequel, dynamic weights (in Laplace domain with the Laplacian $s$ ) are denoted by $W_{\star}$, and ideal transfer function information by $T_{\star}$.

We assume that $n(t)$ can be estimated in real-time via loop detector time-occupancy measurements accurately, $d_{n}(t)$ is scaled by $W_{n}=0.01$ veh. The input flow actuation is penalised on the basis of the maximum allowable entrance flow (based on nominal saturation flow of $1800 \mathrm{veh} / \mathrm{h}$ per lane and maximum green times at 15 signalised junctions located at the perimeter of the network) and hence set to $W_{\beta}=1 / \beta_{\max }$, where $\beta_{\max }=4 \cdot 10^{4}$.

The reference tracking controller is designed with ideal closed loop tracking behaviour given by $T_{r}=1 /\left(\tau_{r} s+1\right)$, where the time constant $\tau_{r}$ is selected on the basis of average travel time in loaded network for $\underline{n} \leq n(t) \leq \bar{n}$, which can be determined from historical traffic data. For the underlying case-study and network $\tau_{r}$ is set equal to $12 \mathrm{~min}$.

Assuming that the reference vehicle accumulation $r(t)=$ $\hat{n}(t)$ is uncertain (due to inappropriate flow capacity estimation in the uncongested and congested regimes of the MFD), 
we introduce uncertainty in the reference signal generation by considering a relative model mismatch with weight $W_{r}$. In case of low frequency changing reference signal, we assume that vehicle accumulation is relatively accurate while it gradually increases for values above the cross-over frequency of $\tau_{r}$. To this end, we select $\sim 10 \%$ uncertainty in case that $r(t)$ operates in the low frequency (uncongested regime) and over $100 \%$ in the high frequency (congested regime) as,

$$
W_{r}=\omega_{r} \cdot \frac{s+1}{s / 150+1}
$$

$\omega_{r}$ can be used to scale uncertainty $\sim 0.1$. Input multiplicative uncertainty is introduced to reflect imperfect control actuation (e.g. delays, saturation flows mismatch, cycle time, control allocation). Relative model uncertainty with $W_{\beta}$ is added to the plant input having similar dynamic properties as $W_{r}$, thus

$$
W_{\beta}=\omega_{\beta} \cdot \frac{1 / 1.5+1}{s / 150+1}
$$

where $\omega_{\beta} \sim 0.1$ is a scaling factor.

In the LPV model (4), MFD parameter mismatch is introduced with coefficients $\delta_{i}, i=1, \ldots, n_{Q}$. It is well known that when the network operating in the congested regime, i.e. $n>\hat{n}$, the MFD parameters vary significantly (cf. the scattered area in the MFD of Fig. 2) unlike in the uncongested regime where the MFD is well-defined. To reflect this anisotropy of $\delta$, we hereby define

$$
W_{\delta_{i}}(p)=c_{i \delta} \cdot \frac{n / \underline{n}}{s \tau_{i \delta}+1}
$$

where $c_{i \delta}$ is the steady state relative uncertainty weight of $c_{i}$ (e.g. 5-10\%). The term $n / \underline{n}$ is normed by the lower polytope bound and applied to mimic gradual parameter variation increase. With $n$, we expresses the frozen parameter value in $\mathcal{P}$ (scheduling dependent weight dynamics). Constant values $\tau_{i \delta}$ are introduced to bound the frequency variation of the uncertainty. These changes of the parameters are usually happens slowly hence we propose $0.5-1 \mathrm{~h}$ as numerical values.

In a similar vein, input flow disturbance $\hat{d}(t)$ in (4) is scaled in frequency domain by an appropriate $\tau_{d}$ in the range of $30 \mathrm{~min}$. Flow disturbances include uncontrolled inflows $d_{\beta}$ at the perimeter of the network. We assume that the uncontrolled inflows at the perimeter encountering higher travel times than the internal flows. Thus, a parameter $d_{0}$ is used to normalise the input disturbance flow,

$$
W_{d}=\frac{d_{0}}{s \tau_{d}+1} .
$$

Finally, the tracking error weight is selected in a way, that for steady-state conditions the effect of disturbance (including the reference signal) is rejected in terms of induced 2 -norm over the tracking error by a factor $\phi(\approx 30-50)$

$$
W_{e}=\phi \cdot \frac{s / 100+1}{s / 0.1+1}
$$

After augmenting the nominal flow dynamics with the aforementioned weights as in Fig. 1, feasible LPV controllers
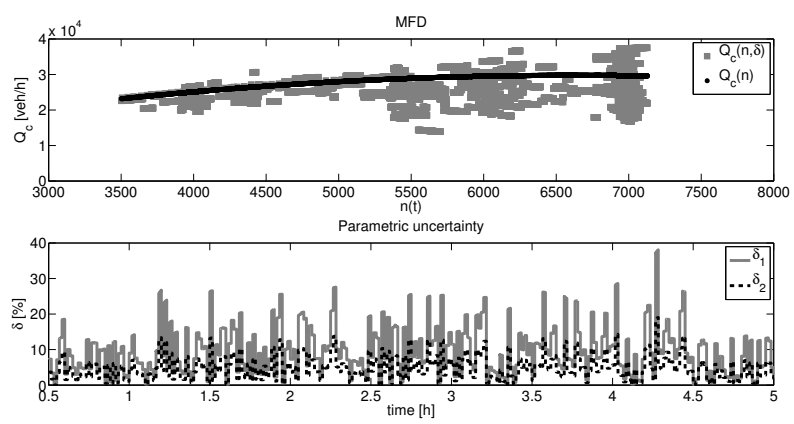

Fig. 3. Parameter uncertainty in MFD. The nominal (black) and uncertain (grey) MFD points are depicted. Furthermore, the relative parameter uncertainty in the MFD coefficients, ranging between $\sim 10-40 \%$ is plotted as $100 \cdot|\delta|$.

have been obtained by the use of the LPVTools [23]. First, LPV controller was derived with a constant Lyapunov function resulting in a disturbance attenuation level $\gamma=36.8$. The performance level has been slightly decreased when using parameter dependent Lyapunov function but closedloop simulation results have shown negligible performance upgrade in time domain.

\section{Simulation Environment and Results}

A macroscopic simulation scenario is set up in MATLAB/SIMULINK environment using the nonlinear equation (1) to mimic the San Francisco case study in Section IVA. Fig. 3 (top plot) depicts a $4.5 \mathrm{~h}$ simulation under both uncongested and congested traffic conditions, i.e. with $n(t) \sim[3500,7000]$. LPV controller design framework with separate control input allocation techniques (presented in the previous Section) are used to track critical accumulation. In the simulation, gated flow $\beta(t)$ is implemented. The flow $\beta(t)$ ordered by (7) is distributed to 15 signalised junctions located at the perimeter of the network, ranging in the interval $\hat{\beta} \sim[1.45,4] \cdot 10^{4}$.

Uncertainty is added to the continuous time flow equation, by abrupting the nominal MFD parameters with relative variations in $\delta_{1}, \delta_{2}\left(n_{Q}=2\right)$. MFD parametric uncertainties are scheduled by $n(t)$, meaning that nominal MFD parameters become more uncertain in congested traffic conditions. Fig. 3 (bottom plot) illustrates the parametric uncertainty values and their effect over the MFD diagram. It can be seen that parameters vary in the range $10-40 \%$, and the uncertainty in parameter $c_{1}$ (reflects the critical accumulation) has been selected to be larger than $c_{2}$ (cf. $\delta_{1}$ with $\delta_{2}$ ). This is because, the maximum output (capacity flow) in the network is a property of the infrastructure and it is less uncertain than the critical accumulation. On the other hand, capacity flow in urban networks may be observed over a range of vehicle-numbers, and thus the critical accumulation cannot accurately be specified [10]. It can be observed in Fig. 3 that parameter mismatches are increasing with $n(t)$. Finally note that the difference between the nominal MFD $Q(n)$ and the uncertain one $\tilde{Q}(n, c, \delta)$ is not symmetric.

Fig. 4 (top plot) shows the optimal control input $\beta(t)$ (grey) calculated by the LPV controller (7). Real-time application of (7) in SIMULINK has been effectuated by 

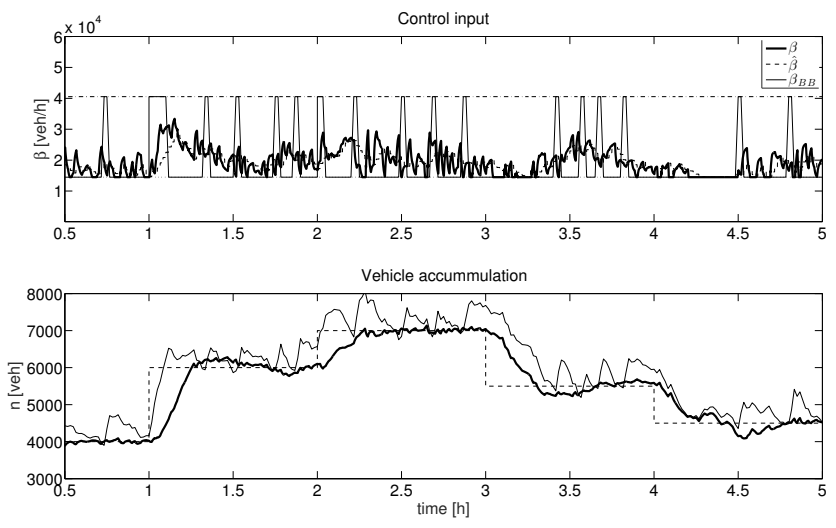

Fig. 4. Optimal ordered flow $\beta(t)$ (solid line) and (real) gated flow $\hat{\beta}(t)$ (dash line) resulting from (8) with $T_{c}=60 \mathrm{~s}$ compared to simple ban-bang control strategy. The bang-bang control algorithm activates the minimum and maximum boundary flow depending on the current measured accumulation is below or above the reference accumulation. System performance is plotted in terms of staircase reference accumulation tracking (black solid line corresponds to robust and grey line to bang-bang control strategy).

means of the (real) gated flow $\hat{\beta}(t)$ resulting from the solution of the optimisation problem (8). The minimum and maximum allowable $\beta(t)$ are highlighted with dotted grey lines. For a fair comparison a reference tracking Bang-Bang (BB) perimeter controller (dash grey line), i.e., with variable critical $\hat{n}(t)$, is simulated and depicted in Fig. 4. The ordered flow $\beta(t)$ is continuously calculated in the background, while $\hat{\beta}(t)$ is only updated every $T_{c i}=60 \mathrm{~s}$.

Fig. 4 (bottom plot) depicts the tracking performance of the proposed LPV controller in a simulation scenario with variable critical reference accumulation $\hat{n}(t)$. In this scenario, critical accumulation is scheduled in the range $[4000,7000]$ veh, while uncertainty in the MFD is introduced according to Fig. 3. The simulated traffic conditions allow the MFD in operating in the uncongested and congested regimes. Clearly, the proposed LPV controller is seen to track smoothly, quickly and quite accurately the actual critical accumulation and reject the modelled uncertainty and disturbances. As can be seen the BB controller is triggered (activated/deactivated) several times during the simulation (top figure) and oscillates between the extremes minimum and maximum. The bottom figure confirms the oscillatory behaviour of the $\mathrm{BB}$ since the flow $\beta_{B B}$ exhibits high-frequency variations over time around $\hat{n}(t)$ (compared to the smooth operation of the LPV).

\section{CONClusion}

Robust perimeter flow control has been proposed in this paper. A LPV model reformulation has been applied to transform the MFD based nonlinear traffic flow dynamics. Uncertainties have been structured in the LPV model framework enabling robust ordered input flow control. Green stages related saturation constraints have been considered in a separate optimisation by means of quadratic and optimal input flow allocation techniques. A macroscopic simulation study has been utilised to demonstrate the applicability, effectiveness and robustness of the proposed strategy.

Future research include application of a robust constrained LPV methodology as indicated in Remark 4 with a superior real-time computation requirement, and validation of the proposed methodology in microscopic simulation.

\section{REFERENCES}

[1] J. S. Shamma and M. Athans, "Guaranteed properties of gain scheduled control for linear parameter-varying plants," Automatica, vol. 27, no. 3 , pp. 559 - 564, 1991.

[2] G. J. Balas, "Linear, parameter-varying control and its application to a turbofan engine," International Journal of Robust Nonlinear Control, vol. 12, pp. 763-796, 2002.

[3] T. Luspay, T. Péni, and B. Kulcsár, "Constrained freeway traffic control via linear parameter varying paradigms," in Control of Linear Parameter Varying Systems With Applications, J. Mohammadpour and C. W. Scherer, Eds. Springer US, 2012, pp. 461-482.

[4] A. Dabiri and B. Kulcsár, "Incident parameter scheduled freeway traffic control - a ramp meter approach," in Proc. 19th IFAC World Congress, 2014, pp. $11338-11343$.

[5] J. W. Godfrey, "The mechanism of a road network," Traffic Engineering and Control, vol. 11, no. 7, pp. 323-327, 1969.

[6] S. Ardekani and R. Herman, "Urban network-wide traffic variables and their relations," Transport Science, vol. 21, no. 1, pp. 1-16, 1987.

[7] C. F. Daganzo and N. Geroliminis, "An analytical approximation for the macroscopic fundamental diagram of urban traffic," Transportation Research Part B, vol. 42, no. 9, pp. 771-781, 2008.

[8] H. Mahmassani, J. Williams, and R. Herman, "Performance of urban traffic networks," in 10th International Symposium on Transportation and Traffic Theory, N. Gartner and N. Wilson, Eds., Elsevier, Amsterdam, The Netherlands, 1987.

[9] N. Geroliminis and C. F. Daganzo, "Existence of urban-scale macroscopic fundamental diagrams: Some experimental findings," Transportation Research Part B, vol. 42, no. 9, pp. 759-770, 2008.

[10] K. Ampountolas and A. Kouvelas, "Real-time estimation of critical vehicle accumulation for maximum network throughput," in Proc. 2015 American Control Conference, Chicago, IL, July 1-3, 2015.

[11] C. F. Daganzo, "Urban gridlock: Macroscopic modeling and mitigation approaches," Transport Res Part B, vol. 41, no. 1, pp. 49-62, 2007.

[12] M. Keyvan-Ekbatani, A. Kouvelas, I. Papamichail, and M. Papageorgiou, "Exploiting the fundamental diagram of urban networks for feedback-based gating," Transport Res Part B, vol. 46, no. 10, pp. 1393-1403, 2012.

[13] J. Haddad and A. Shraiber, "Robust perimeter control design for an urban region," Transport Res Part B, vol. 68, pp. 315-332, 2014.

[14] J. Haddad and N. Geroliminis, "On the stability of traffic perimeter control in two-region urban cities," Transportation Research Part B, vol. 46, no. 9, pp. 1159-1176, 2012.

[15] K. Aboudolas and N. Geroliminis, "Perimeter and boundary flow control in multi-reservoir heterogeneous networks," Transportation Research Part B, vol. 55, pp. 265-281, 2013.

[16] N. Geroliminis, J. Haddad, and M. Ramezani, "Optimal perimeter control for two urban regions with macroscopic fundamental diagrams: A model predictive approach," IEEE Trans Intell Transport Syst, vol. 14, no. 1, pp. 348-359, 2013.

[17] A. Mazloumian, N. Geroliminis, and D. Helbing, "The spatial variability of vehicle densities as determinant of urban network capacity," Phil Trans Royal Society A, vol. 368, no. 1928, pp. 4627-4647, 2010.

[18] K. Aboudolas, M. Papageorgiou, A. Kouvelas, and E. Kosmatopoulos, "A rolling-horizon quadratic-programming approach to the signal control problem in large-scale congested urban road networks," Transportation Research Part C, vol. 18, no. 5, pp. 680-694, 2010.

[19] T. Tettamanti, T. Luspay, B. Kulcsar, T. Peni, and I. Varga, "Robust control for urban road traffic networks," IEEE Transactions on Intelligent Transportation Systems, vol. 15, no. 1, pp. 385-398, 2014.

[20] N. Geroliminis, N. Zheng, and K. Ampountolas, "A three-dimensional macroscopic fundamental diagram for mixed bi-modal urban networks," Transportation Research Part C, vol. 42, pp. 168-181, 2014.

[21] C. Buisson and C. Ladier, "Exploring the impact of homogeneity of traffic measurements on the existence of macroscopic fundamental diagrams," Transport Res Rec, no. 2124, pp. 127-136, 2009.

[22] F. Wu, X. Yang, A. Packard, and G. Becker, "Induced L2-norm control for LPV systems with bounded parameter variation rates," Int Journal of Robust and Nonlinear Control, vol. 6, pp. 983-998, 1996.

[23] A. Hjartarson, P. Seiler, and G. Balas, "LPV aeroservoelastic control using the LPVTools toolbox," 2013.

[24] T. A. Johansen and T. I. Fossen, "Control allocation - A survey," Automatica, vol. 49, no. 5, pp. 1087 - 1103, 2013. 